\title{
Efecto de los inotrópicos sobre la mortalidad en falla cardiaca aguda. Metaanálisis en red de ensayos clínicos
}

\author{
Juan M. Sénior ${ }^{\mathrm{a}, \mathrm{b}, \mathrm{c}, *}$, Edison Muñoz ${ }^{\mathrm{b}, \mathrm{c}}$ y James Díaz ${ }^{\mathrm{c}}$ \\ a Posgrado Cardiología Clínica y Cardiología Intervencionista, Universidad de Antioquia, Medellín, Colombia \\ ${ }^{\mathrm{b}}$ Hospital Universitario San Vicente Fundación, Medellín, Colombia \\ c Grupo para la Investigación de Enfermedades Cardiovasculares, Sección Cardiología, Departamento de Medicina Interna, \\ Universidad de Antioquia, Medellín, Colombia
}

Recibido el 22 de octubre de 2016; aceptado el 31 de enero de 2017

Disponible en Internet el 16 de mayo de 2017

\section{PALABRAS CLAVE \\ Falla cardiaca; \\ Agentes inotrópicos; \\ Metaanálisis}

\begin{abstract}
Resumen
Introducción: en pacientes con falla cardiaca aguda es necesario el uso de inotrópicos para lograr su estabilización.

Objetivo: definir cuál de los medicamentos inotrópicos se asocia con menor mortalidad.

Metodología: se realizó un metaanálisis en red con la aproximación frecuentista. La búsqueda sistemática incluyó PUBMED, EMBASE, CENTRAL, DARE, Epistemonikos, SieELO, LILACS y OpenGray. Se incluyeron ensayos clínicos con asignación aleatoria en pacientes con falla cardiaca aguda que recibieron dobutamina, levosimendán o milrinone que reportaran datos de mortalidad.

Resultados: los 20 estudios incluidos asignaron en forma aleatoria 5.315 pacientes a cinco comparaciones. La mayoría de estudios mostró riesgo de sesgos de selección de la población y en el cegamiento de la intervención. Las diferentes comparaciones con placebo, dobutamina (OR 1,30 IP 95\% 0,54-3,09), levosimendán (OR 0,86 IP 95\% 0,38-1,93) y milrinone (OR 1,69 IP 95\% $0,49-5,85)$, no mostraron diferencias estadísticamente significativas, al igual que las comparaciones entre ellos, dobutamina vs. levosimendán (OR 0,66 IP 95\% 0,30-1,48), dobutamina vs. milrinone (OR 1,30 IP 95\% 0,34-4,96) y levosimendán vs. milrinone (OR 1,97 IP 95\% 0,53-7,29). El análisis por rangos demostró mayor probabilidad para milrinone seguido de dobutamina.

Discusión: no hay diferencias en el uso de inotrópicos como dobutamina, levosimendán o milrinone en términos del desenlace de mortalidad, así que su utilización dependerá de otras consideraciones.

(C) 2017 Sociedad Colombiana de Cardiología y Cirugía Cardiovascular. Publicado por Elsevier España, S.L.U. Este es un artículo Open Access bajo la licencia CC BY-NC-ND (http:// creativecommons.org/licenses/by-nc-nd/4.0/).
\end{abstract}

\footnotetext{
* Autor para correspondencia.

Correo electrónico: mmbt@une.net.co (J.M. Sénior).
} 


\section{KEYWORDS}

Heart failure; Inotropes;

Meta-analysis
Effect of inotropic drugs on mortality in acute heart failure. Network meta-analysis of clinical trials

\begin{abstract}
Introduction: Inotropic drugs are required in order to stabilise patients with acute heart failure. Objective: To determine which of the inotropic drugs are associated with lower mortality.

Materials and method: A network meta-analysis was performed using the frequentist approach. The systematic search included PUBMED, EMBASE, CENTRAL, DARE, Epistemonikos, ScieELO, LILACS, and OpenGray data bases. Randomised clinical trials were included that provided data on mortality on patients with acute heart failure who received dobutamine, levosimendan or milrinone.

Results: A total of 5,315 patients in the 20 studies include were randomly assigned to five comparisons. The majority of the studies showed bias risks in population selection and in the blinding of the intervention. The different comparisons with placebo, dobutamine (OR 1.30; 95\% probability interval (PI); 0.54-3.09), levosimendan (OR 0.86; 95\% PI; 0.38-1.93), and milrinone (OR 1.69; 95\% PI; 0.49-5.85), did not show any statistically significant differences. The same applied to the comparisons between themselves, dobutamine vs. levosimendan (OR 0.66; 95\% PI; 0.30-1.48), dobutamine vs. milrinone (OR 1.30; 95\% PI; 0.34-4.96), and levosimendan vs. milrinone (OR 1.97; 95\% PI; 0.53-7.29). The analysis by ranges showed a higher probability for milrinone, followed by dobutamine.

Discussion: There are no differences in the use of inotropic drugs, such as dobutamine, levosimendan or milrinone in terms of mortality outcomes. Their use, therefore, will depend on other factors.

(c) 2017 Sociedad Colombiana de Cardiología y Cirugía Cardiovascular. Published by Elsevier España, S.L.U. This is an open access article under the CC BY-NC-ND license (http:// creativecommons.org/licenses/by-nc-nd/4.0/).
\end{abstract}

\section{Introducción}

La falla cardiaca aguda se define como el inicio rápido o empeoramiento gradual del estado clínico que requiere evaluación y tratamiento urgente, generalmente hospitalario ${ }^{1}$. Desde el punto de vista clínico, incluye tres escenarios diferentes, por lo que puede clasificarse como empeoramiento de una falla cardiaca crónica (70\%), falla cardiaca de novo o falla cardiaca avanzada con choque cardiogénico ${ }^{2}$. Las manifestaciones clínicas pueden variar en forma importante en un espectro que incluye desde formas leves en las que predomina la hipertensión, pasando por sobrecarga de volumen con o sin edema pulmonar, hasta cuadros más severos con choque cardiogénico, que por fortuna representan el $1 \%^{3}$; a pesar de esto, algunos pacientes pueden presentar signos de hipoperfusión con o sin hipotensión asociada y requerir el uso de inotrópicos hasta en el $19 \%$ de los casos, como lo demostró el registro ADHERE (Acute Decompensated Heart Failure National Registry) ${ }^{4}$.

El uso de inotrópicos no ha estado exento de controversia, puesto que se les relaciona con aumento de arritmias ventriculares y mortalidad a pesar de mejorar el estado hemodinámico y los síntomas ${ }^{5}$; sin embargo, está claro que son necesarios para lograr la estabilización en ese grupo de pacientes y posteriormente definir la introducción de medicamentos que cambian el pronóstico a largo plazo. Entre los medicamentos inotrópicos se dispone de la dobutamina, que es una amina simpáticomimética, y el milrinone, que es un inhibidor de fosfodiesterasa $3^{6}$; en la última década han aparecido medicamentos inodilatadores potentes, representados básicamente por el levosimendán, el cual sensibiliza las proteínas contráctiles miocárdicas al calcio, como mecanismo de acción diferenciador ${ }^{7}$.

Aunque se han publicado diversos estudios en los que se avala su utilización, generalmente los diseños son inadecuados por antiguos, con pocos ensayos clínicos con asignación aleatoria (ECA), comparaciones con placebo o con solo dos medicamentos, uso de desenlaces subrogados o intermedios, diversos escenarios clínicos, publicaciones incompletas de estos con solo versiones en resumen y resultados contradictorios. Por ende, los metaanálisis del tema poseen los mismos problemas si no se realizan en forma adecuada y no se establecen comparaciones claras entre los medicamentos comercialmente disponibles a los cuales se enfrenta el clínico ${ }^{8}$. El metaanálisis en red es una técnica estadística que permite hacer un estimativo global de la comparación de la efectividad de diversos tratamientos combinando evidencia directa e indirecta; es decir, permite realizar comparaciones no hechas en $\mathrm{ECA}^{9,10}$, de modo que se convierte en una herramienta útil en este escenario.

Dentro de la convocatoria 637-2012 del Departamento Administrativo de Ciencia, Tecnología e Innovación (Colciencias) se desarrolló la Guía de Práctica Clínica para la prevención, diagnóstico, tratamiento y rehabilitación de la falla cardíaca en población mayor de 18 años clasificación $B$, $C$ y $D$, en una de cuyas preguntas fue necesario realizar un metaanálisis en red de los estudios clínicos con asignación aleatoria, con el objetivo de comparar la morbilidad y mortalidad con el uso de dobutamina, levosimendán y milrinone para el tratamiento de la falla cardiaca aguda. 


\section{Materiales y métodos}

El diseño del estudio es de revisión sistemática y metaanálisis en red, cuyo reporte se ajusta a las directrices de Preferred Reporting Items for Systematic Reviews and MetaAnalysis (PRISMA) statement (PRISMA-NMA) ${ }^{11,12}$

\section{Objetivo}

La pregunta PICO precisamente guarda ese orden pacienteintervención-comparador-desenlace; en la forma en que aparece queda desenlace-intervención-comparadorpaciente. No cambia el sentido, pero debería dejarse como el original.

\section{Criterios de elegibilidad}

La selección de los estudios fue realizada por dos investigadores y las diferencias se resolvieron por consenso o con el concurso de un tercero; se incluyeron ensayos clínicos con asignación aleatoria que compararan el uso intravenoso de los inotrópicos dobutamina, levosimendán, milrinone con placebo o entre ellos, y que reportaran datos de mortalidad, estancia hospitalaria y complicaciones relacionadas con el uso de los medicamentos en estudio. No hubo limitación por estado de publicación (resumen o completo) ni por fecha (hasta enero 2015) ni idioma. Se excluyeron estudios con enoximone porque no está disponible comercialmente en el país. Se envió comunicación a los autores del estudio CASINO para conocer los resultados completos, puesto que solo se dispone de publicación como resumen, a pesar de ser un estudio antiguo; no se obtuvo respuesta. Se extrajo de cada estudio el número de pacientes participantes, el número de muertes en cada grupo de estudio, estancia hospitalaria cuando fue reportada y desenlaces de seguridad como presencia de arritmias y estado hemodinámico, entre otros. El desenlace primario evaluado fue mortalidad.

\section{Estrategia de búsqueda}

Se realizó una búsqueda sistemática en las bases de datos bibliográficas PUBMED, EMBASE, CENTRAL, DARE, Epistemonikos, SciELO, LILACS y OpenGray; además, se consultaron páginas web de registros de ensayos clínicos como ClinicalTrials. La búsqueda manual incluyó páginas de congresos internacionales de Cardiología como el de la European Society of Cardiology, American Heart Association y American College of Cardiology, así como los estudios primarios incluidos en las guías de práctica clínica de esas sociedades y de la National Institute for Health and Care Excellence (NICE) y meta-análisis.

La búsqueda sistemática estuvo a cargo de una bibliotecóloga con entrenamiento en el centro Cochrane en España y la estrategia incluyó los siguientes términos: "Heart Failure"'[MeSH] OR "acute heart failure"'[tiab] OR AHF[tiab]) AND (levosimendán[tiab] OR simendan[tiab] OR dextrosimendan[tiab] OR Milrinone[MeSH] OR milrinone[tiab] OR Dobutamine[MeSH] OR dobutamin*[tiab])) AND systematic[sb]) OR ((("Heart Failure"'[MeSH] OR "acute heart failure"'[tiab] OR AHF[tiab]) AND (levosimen-

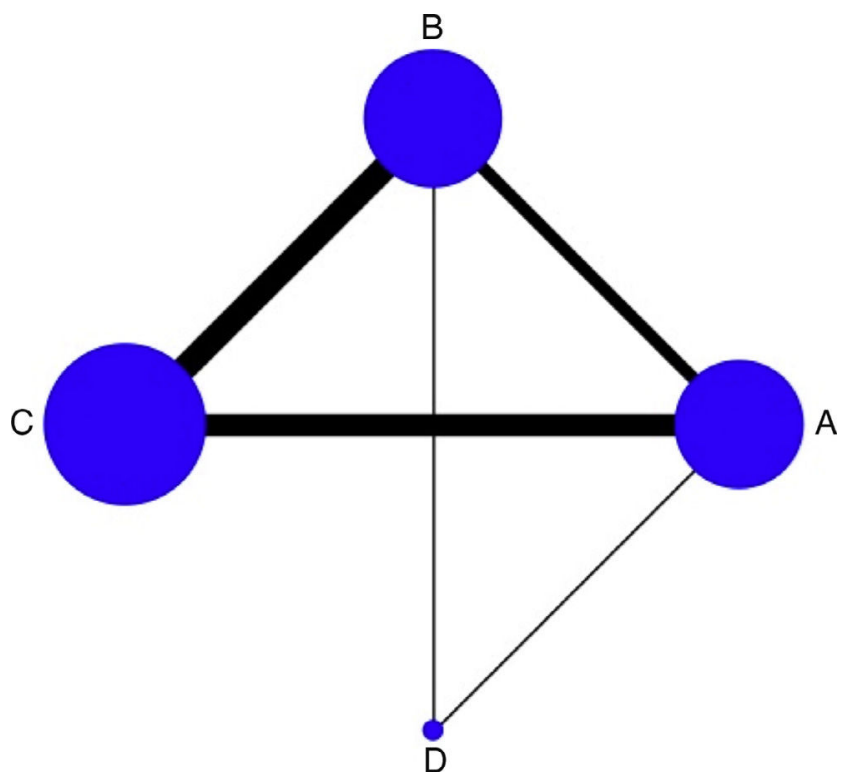

Figura 1 Representación gráfica de los estudios incluidos en el metaanálisis. $A$ = placebo; $B=$ dobutamina; $C=$ levosimendán; $\mathrm{D}=$ milrinone.

dán[tiab] OR simendan[tiab] OR dextrosimendan[tiab] OR Milrinone[MeSH] OR milrinone[tiab] OR Dobutamine[MeSH] OR dobutamin*[tiab])) AND (randomized controlled trial[pt] OR controlled clinical trial[pt] OR randomized[tiab] OR placebo[tiab] OR drug therapy[sh] OR randomly[tiab] OR trial[tiab] OR groups[tiab]) NOT (animals[mh] NOT humans[mh]"'.

\section{Análisis estadístico}

Las comparaciones entre los tratamientos se visualizan por medio de una gráfica estándar que expresa la geometría de la red, en la cual se observa asimetría por la escasa representación de estudios con milrinone (fig. 1). Se realizó un metaanálisis multivariante con el objetivo de sintetizar en forma conjunta los resultados, tanto de las comparaciones directas (versus placebo o cabeza a cabeza), como indirectas (a través de un nodo) y mixtas (corroborando si existe inconsistencia), presentadas como diagrama de bosque (Forrest Plot) con medida de resumen como OR (odds ratio) por efectos aleatorios, utilizando el log OR, con sus respectivos intervalos de confianza y de predicción, y una tabla de resumen; los estudios con cero eventos son artificialmente incluidos al adicionar 0,5 . Se evaluó la heterogeneidad clínica o transitividad con base en las características de cada uno de los estudios y la población incluida, y la heterogeneidad estadística por medio de la inconsistencia con una aproximación local por método de Bucher y global por modelo de inconsistencia para toda la red con $C h i^{2}$ y $p>0,05$. Adicionalmente, como medida que refleja la clasificación de los diversos tratamientos se utilizó la probabilidad de clasificación acumulada (SUCRA: Surface Under the Cumulative RAnking curve); esta medida permite establecer la probabilidad de que una intervención sea la mejor y es expresada en porcentaje. Todos los análisis fueron hechos con el paquete estadístico STATA 13. 


\section{Resultados}

La búsqueda sistemática recuperó 593 referencias y la búsqueda manual en los estudios referenciados en las guías del American College of Cardiology (ACC)/American Heart Association (AHA) del 2013, las guías de la European Society of Cardiology (ESC) de 2012, y las guías del National Institute for Health and Care Excellence (NICE) 2014, donde se encontraron dos referencias adicionales que no aparecían en la búsqueda sistemática. Además, en las referencias de los artículos de revisión se identificaron otras 18 referencias. Después de la revisión de títulos y resúmenes se identificaron 20 estudios primarios de los diferentes inotrópicos a evaluar, que cumplían los criterios de inclusión (fig. 2).

Los 20 estudios incluidos en el análisis final aleatorizaron 5.315 pacientes a grupos de tratamientos así: levosimendán versus placebo (\#9) y levosimendán versus dobutamina (\#10); dobutamina versus placebo (\#5) y dobutamina versus milrinone (\#1); milrinone versus placebo (\#1). Tres estudios asignaron en forma aleatoria pacientes a tres grupos ${ }^{13-15}$, mientras que el resto solo compararon dos grupos ${ }^{16-32}$. El comparador más frecuente fue el levosimendán seguido de la dobutamina. El seguimiento varía entre los estudios. Las características principales se describen en la tabla 1.

En conjunto, los estudios incluidos demuestran heterogeneidad clínica, aunque la mayoría se centra en pacientes con falla cardiaca descompensada, con fracción de eyección reducida y estado funcional NYHA III-IV (New York Heart Association), lo que podría asegurar el supuesto de transitividad; sin embargo, en algunos de ellos las dosis de los medicamentos varían, al igual que el tiempo de infusión, la evaluación de los parámetros hemodinámicos y su consideración para cambiar la dosis (aumentar o disminuir), el tiempo de seguimiento al cual se definía el desenlace, y por último, que la estrategia fuese considerada como utilización de rutina (particularmente en el estudio OPTIME-CHF) o de acuerdo con variables que sugirieran la necesidad del inotrópico.

Los estudios incluidos son de moderado a alto riesgo de sesgos según la recomendación Cochrane, resaltando que muchos de los ítems fueron calificados como No Claro, a excepción de los estudios LEAF, LIDO y SURVIVE. En 15 de ellos [(Nieminen (2000), Packer (2003 y 2013) (REVIVE I), Kivikko (2003), Colettaa (2004), Clelanda (2004), Zairis (2004) - CASINO, Cleland (2006 y Packer (2013) (REVIVE ॥), Adamopoulos (2006), Flevari (2006), Follath (1999), Samimi (2008), Duygu (2007), Duygu (2008), Bergh (2010), Liang (2014), Bader (2010) y Aranda (2003)] no fue claro el mecanismo para la asignación aleatoria. El ocultamiento de la asignación presentó alto riesgo de sesgo en 4 estudios [(Samimi (2008), Duygu (2007), Bader (2010) y Aranda (2003)], bajo riesgo de sesgo en 4 [(Husebye (2013) LEAF, Follath (2002) LIDO, Mebazaa (2007) SURVIVE y Cuffe (2002) OPTIME)] y no fue claro en los demás. El enmascaramiento de los participantes presentó bajo riesgo de sesgos en 4 estudios [(Husebye (2013) LEAF, Follath (2002) LIDO, Mebazaa (2007) SURVIVE y Cuffe (2002) OPTIME)] y en los demás no estaba descrito, pero por tratarse del desenlace mortalidad se calificó como no claro. No hubo estudios con desenlaces incompletos, pero sí 4 estudios con alto riesgo de reporte

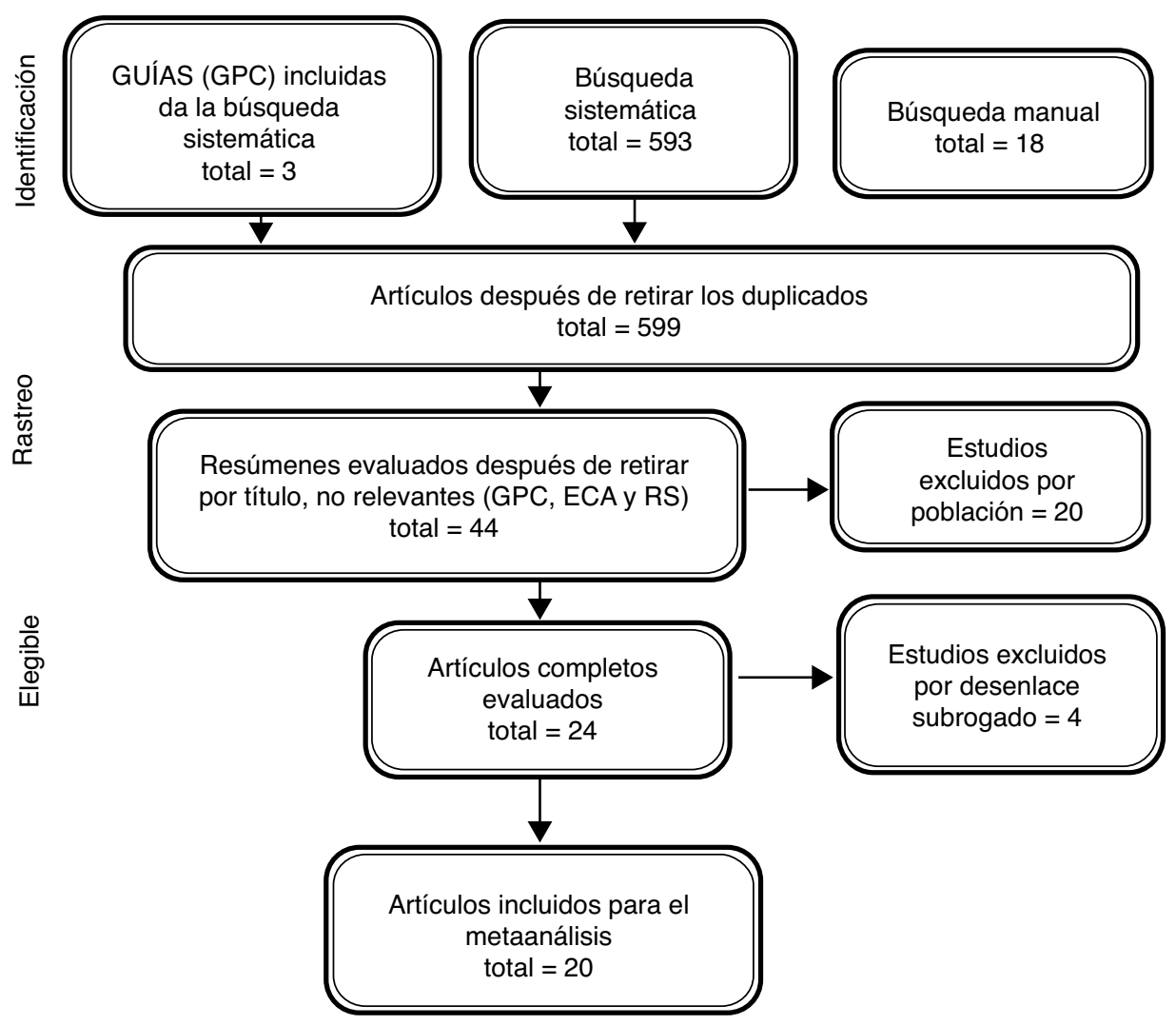

Figura 2 Flujograma de búsqueda. 
Tabla 1 Características de los estudios incluidos

\begin{tabular}{|c|c|c|c|c|c|c|c|}
\hline Estudio & Año & Población & Intervención & Comparación & & $\begin{array}{l}\text { Tiempo de } \\
\text { seguimiento }\end{array}$ & Muerte \\
\hline Nieminen & 2000 & $\begin{array}{l}\text { Falla cardiaca II a Iv y } \\
\text { FEVI }<40 \%(n=151)\end{array}$ & $\begin{array}{l}\text { Diferentes dosis de } \\
\text { levosimendán }(n=95)\end{array}$ & $\begin{array}{l}\text { Dobutamina a } 6 \\
\mathrm{mcg} / \mathrm{kg} / \mathrm{min} \sin \\
\text { carga }(\mathrm{n}=20)\end{array}$ & Placebo $(n=36)$ & NR & $\begin{array}{l}\text { Levosimendán }=1, \\
\text { Dobutamina }=1, \\
\text { Placebo }=0\end{array}$ \\
\hline RUSSLAN & 2002 & $\begin{array}{l}\text { Pacientes con falla } \\
\text { cardiaca aguda } \\
\text { posinfarto y necesidad } \\
\text { de inotrópicos }(n=504)\end{array}$ & $\begin{array}{l}\text { Levosimendán a diferentes } \\
\text { dosis }(n=402)\end{array}$ & Placebo $(n=102)$ & & Muerte $=6$ meses & $\begin{array}{l}\text { Levosimendán = 91, } \\
\text { Placebo }=32\end{array}$ \\
\hline REVIVE । & 2003 & $\begin{array}{l}\text { Falla cardiaca } \\
\text { descompensada }(n=100 \\
\text { pacientes) }\end{array}$ & $\begin{array}{l}\text { Levosimendán } 12 \mathrm{mcg} / \mathrm{kg} \text { en } \\
\text { bolo, infusión a } 0,1 \\
\mathrm{mcg} / \mathrm{kg} / \mathrm{min} \text { por } 50 \text { minutos } \\
\text { y si toleraba a } 0,2 \\
\mathrm{mcg} / \mathrm{kg} / \mathrm{min} \text { por } 23 \text { horas } \\
\text { más }(n=51)\end{array}$ & Placebo $(n=49)$ & & Muerte a 14 días & $\begin{array}{l}\text { Levosimendán }=4 \\
\text { Placebo }=5\end{array}$ \\
\hline Kivikko & 2003 & $\begin{array}{l}\text { Pacientes con falla } \\
\text { cardiaca NYHA III O IV y } \\
\text { FEVI <30\% } \\
\text { descompensada }(n=146)\end{array}$ & $\begin{array}{l}\text { Levosimendán por hasta } 48 \\
\text { horas }(n=98)\end{array}$ & Placebo $(n=48)$ & & $\begin{array}{l}\text { Muerte = } 14 \text { días, } \\
\text { Hemodinámi- } \\
\text { cas }=24 \\
\text { horas }\end{array}$ & $\begin{array}{l}\text { Levosimendán }=2 \\
\text { Placebo }=3\end{array}$ \\
\hline CASINO & 2004 & $\begin{array}{l}\text { Falla cardiaca NYHA IV, } \\
\text { FEVI }<35 \%(n=299)\end{array}$ & Levosimendán $(n=100)$ & $\begin{array}{l}\text { Dobutamina, dosis } \\
\text { no publicada } \\
(n=100)\end{array}$ & Placebo $(n=99)$ & Muerte a 6 meses & $\begin{array}{l}\text { Levosimendán }=18 \\
\text { Dobutamina }=42 \\
\text { Placebo }=28\end{array}$ \\
\hline REVIVE ॥ & 2006 & $\begin{array}{l}\text { Pacientes con FEVI }<35 \% \\
\text { y disnea a pesar de } \\
\text { diuréticos y } \\
\text { vasodilatadores }\end{array}$ & $\begin{array}{l}\text { Levosimendán } 12 \mathrm{mcg} / \mathrm{kg} \text { en } \\
\text { bolo, infusión a } 0,1 \\
\mathrm{mcg} / \mathrm{kg} / \mathrm{min} \text { por } 50 \text { minutos } \\
\text { y si toleraba a } 0,2 \\
\mathrm{mcg} / \mathrm{kg} / \mathrm{min} \text { por } 23 \text { horas } \\
\text { más }(\mathrm{n}=299)\end{array}$ & Placebo $(n=301)$ & & $\begin{array}{l}\text { Muerte a } 90 \text { días, } \\
\text { concentración de } \\
\text { BNP a las } 24 \text { h y } 5 \\
\text { días }\end{array}$ & $\begin{array}{l}\text { Levosimendán }=45 \\
\text { Placebo }=35\end{array}$ \\
\hline Adamopoulos & 2006 & $\begin{array}{l}\text { NYHA III/Iv, admitidos por } \\
\text { descompensación. FEVI } \\
<30 \%\end{array}$ & $\begin{array}{l}\text { Levosimendán } 6 \mathrm{mcg} / \mathrm{kg} \text { en } \\
\text { bolo e infusión de } 0,1 \\
\mathrm{mcg} / \mathrm{kg} / \mathrm{min} 24 \text { horas } \\
(\mathrm{n}=23)\end{array}$ & $\begin{array}{l}\text { Dobutamina a } 5 \\
\mathrm{mcg} / \mathrm{kg} / \mathrm{min} \text { y } \\
\text { aumento en caso } \\
\text { de no mejoría } \\
(\mathrm{n}=23)\end{array}$ & Placebo $(n=23)$ & $\begin{array}{l}\text { Muerte }=4 \text { meses, } \\
\text { Hemodinámicas }=3 \\
\text { días }\end{array}$ & $\begin{array}{l}\text { Levosimendán }=2, \\
\text { Dobutamina }=5 \\
\text { Placebo }=4\end{array}$ \\
\hline Flevari & 2006 & $\begin{array}{l}\text { Hospitalizados por falla } \\
\text { cardiaca NYHA III O IV y } \\
\text { FEVI }<30 \%(n=45)\end{array}$ & $\begin{array}{l}\text { Levosimendán sin bolo, a } \\
0,1 \mathrm{mcg} / \mathrm{kg} / \mathrm{min} 24 \text { horas } \\
(\mathrm{n}=30)\end{array}$ & Placebo $(n=15)$ & & Muerte $=3$ meses & $\begin{array}{l}\text { Levosimendán = } 3 \\
\text { Placebo }=1\end{array}$ \\
\hline
\end{tabular}




\begin{tabular}{|c|c|c|c|c|c|c|}
\hline Estudio & Año & Población & Intervención & Comparación & $\begin{array}{l}\text { Tiempo de } \\
\text { seguimiento }\end{array}$ & Muerte \\
\hline LEAF & 2013 & $\begin{array}{l}\text { Pacientes pos IAM con ST } \\
\text { con signos de falla } \\
\text { cardiaca }(61)\end{array}$ & $\begin{array}{l}\text { Levosimendán } 0,2 \\
\mathrm{mcg} / \mathrm{kg} / \mathrm{min} 1 \text { hora seguido } \\
\text { a } 0,1 \mathrm{mcg} / \mathrm{kg} / \mathrm{min} 24 \text { horas } \\
(\mathrm{n}=30)\end{array}$ & Placebo $(n=31)$ & Muerte $=6$ meses & $\begin{array}{l}\text { Levosimendán }=173 \\
\text { Dobutamina }=185\end{array}$ \\
\hline Follath & 1999 & $\begin{array}{l}\text { Choque cardiogénico o } \\
\text { falla cardiaca con bajo } \\
\text { gasto }(n=19)\end{array}$ & $\begin{array}{l}\text { Levosimendán bolo } 12 \\
\mathrm{mcg} / \mathrm{kg} \text { seguido infusión a } \\
0,2 \mathrm{mcg} / \mathrm{kg} / \mathrm{min} \text {, con } \\
\text { posibilidad de segundo bolo } \\
\text { si no había mejoría del } \\
\text { índice cardiaco }(\mathrm{n}=9)\end{array}$ & $\begin{array}{l}\text { Dobutamina a } 8 \mathrm{mcg} / \mathrm{kg} / \mathrm{min}, \text { y aumento si } \\
\text { no mejoraba índice cardiaco }(\mathrm{n}=10)\end{array}$ & NR & $\begin{array}{l}\text { Muerte } \\
\text { Levosimendán = } 3 \\
\text { Dobutamina }=3\end{array}$ \\
\hline LIDO & 2002 & $\begin{array}{l}\text { Falla cardiaca con FEVI } \\
<35 \% \text { y bajo gasto } \\
(n=203)\end{array}$ & $\begin{array}{l}\text { Levosimendán bolo } 24 \\
\mathrm{mcg} / \mathrm{kg} \text {, seguido a } 0,1 \\
\mathrm{mcg} / \mathrm{kg} / \mathrm{min} 24 \text { horas } \\
(\mathrm{n}=103)\end{array}$ & $\begin{array}{l}\text { Dobutamina a } 5 \mathrm{mcg} / \mathrm{kg} / \mathrm{min} \text { sin carga } \\
(\mathrm{n}=100)\end{array}$ & 180 días & $\begin{array}{l}\text { Levosimendán }=27 \\
\text { Dobutamina }=38\end{array}$ \\
\hline Samimi-Fard & 2007 & $\begin{array}{l}\text { Pacientes con choque } \\
\text { posIAM (22) }\end{array}$ & $\begin{array}{l}\text { Levosimendán bolo } 24 \\
\mathrm{mcg} / \mathrm{kg} \text { seguido a } 0,1 \\
\mathrm{mcg} / \mathrm{kg} / \mathrm{min} 24 \text { horas } \\
(\mathrm{n}=11)\end{array}$ & $\begin{array}{l}\text { Dobutamina a } 5 \\
\mathrm{mcg} / \mathrm{kg} / \mathrm{min} \sin \\
\text { carga }(\mathrm{n}=11)\end{array}$ & 20 meses & $\begin{array}{l}\text { Levosimendán }=3 \\
\text { Dobutamina }=1\end{array}$ \\
\hline Duygu & 2007 & $\begin{array}{l}\text { Falla cardiaca } \\
\text { isquémica, con FEVI } \\
<40 \% \text { y NYHA III y IV } \\
(n=60)\end{array}$ & $\begin{array}{l}\text { Levosimendán bolo } 6-12 \\
\mathrm{mcg} / \mathrm{kg} \text { seguido a } 0,1 \\
\mathrm{mcg} / \mathrm{kg} / \mathrm{min} 24 \text { horas } \\
(\mathrm{n}=30)\end{array}$ & $\begin{array}{l}\text { Dobutamina a } 5 \mathrm{mcg} / \mathrm{kg} / \mathrm{min} \text { y aumento } \\
\text { hasta } 20 \mathrm{mcg} / \mathrm{kg} / \mathrm{min}(\mathrm{n}=30)\end{array}$ & NR & $\begin{array}{l}\text { Muerte } \\
\text { intrahospitalaria } \\
\text { Levosimendán = } 2 \\
\text { Dobutamina }=1\end{array}$ \\
\hline SURVIVE & 2007 & $\begin{array}{l}\text { Falla cardiaca } \\
\text { descompensada, FEVI } \\
<30 \% \text { y necesidad de } \\
\text { inotrópicos }(n=1327)\end{array}$ & $\begin{array}{l}\text { Levosimendán carga de } 12 \\
\mathrm{mcg} / \mathrm{kg} \text { e infusión a } 0,1 \\
\mathrm{mcg} / \mathrm{kg} / \mathrm{min} 24 \text { horas } \\
(\mathrm{n}=664)\end{array}$ & $\begin{array}{l}\text { Dobutamina a } 5 \mathrm{mcg} / \mathrm{kg} / \mathrm{min} \text { y aumento } \\
\text { hasta } 40 \mathrm{mcg} / \mathrm{kg} / \mathrm{min}(\mathrm{n}=663)\end{array}$ & 180 días & $\begin{array}{l}\text { Muerte Levosimen- } \\
\text { dán }=173 \\
\text { Dobutamina }=185\end{array}$ \\
\hline Duygu & 2008 & $\begin{array}{l}\text { Falla cardiaca } \\
\text { isquémica, con FEVI } \\
<40 \% \text { y NYHA III y IV, que } \\
\text { tomaran carvedilol } \\
(n=40)\end{array}$ & $\begin{array}{l}\text { Levosimendán bolo } 6-12 \\
\mathrm{mcg} / \mathrm{kg} \text { seguido a } 0,1 \\
\mathrm{mcg} / \mathrm{kg} / \mathrm{min} 24 \text { horas } \\
(\mathrm{n}=20)\end{array}$ & $\begin{array}{l}\text { Dobutamina a } 5 \mathrm{mcg} / \mathrm{kg} / \mathrm{min} \text { y aumento } \\
\text { hasta } 20 \mathrm{mcg} / \mathrm{kg} / \mathrm{min}(\mathrm{n}=20)\end{array}$ & 30 días & $\begin{array}{l}\text { Levosimendán = } 1 \\
\text { Dobutamina }=2\end{array}$ \\
\hline
\end{tabular}




\begin{tabular}{|c|c|c|c|c|c|c|}
\hline Estudio & Año & Población & Intervención & Comparación & $\begin{array}{l}\text { Tiempo de } \\
\text { seguimiento }\end{array}$ & Muerte \\
\hline Bergh & 2009 & $\begin{array}{l}\text { Falla cardiaca con FEVI } \\
<35 \% \text { y NYHA III y IV } \\
(n=60)\end{array}$ & $\begin{array}{l}\text { Levosimendán } 12 \mathrm{mcg} / \mathrm{kg} \text { en } \\
\text { bolo seguido por infusión de } \\
0,1 \text { a } 0,2 \mathrm{mcg} / \mathrm{kg} / \mathrm{min} \text { por } \\
24 \text { horas }(\mathrm{n}=29)\end{array}$ & $\begin{array}{l}\text { Dobutamina a } 5 \mathrm{mcg} / \mathrm{kg} / \mathrm{min} \text { y aumento } \\
\text { hasta } 10 \mathrm{mcg} / \mathrm{kg} / \mathrm{min} \text { por } 47 \text { horas }(\mathrm{n}=31)\end{array}$ & 30 días & $\begin{array}{l}\text { Levosimendán }=2 \\
\text { Dobutamina }=1\end{array}$ \\
\hline Liang & 1984 & $\begin{array}{l}\text { Falla cardiaca II a Iv } \\
(n=15)\end{array}$ & $\begin{array}{l}\text { Dobutamina a } 5-25 \\
\mathrm{mcg} / \mathrm{kg} / \mathrm{min} \text { por } 72 \text { horas } \\
(\mathrm{n}=8)\end{array}$ & Placebo $(n=7)$ & 4 semanas & $\begin{array}{l}\text { Dobutamina }=0 \\
\text { Placebo }=0\end{array}$ \\
\hline OPTIME-CHF & 2002 & $\begin{array}{l}\text { Descompensación de } \\
\text { falla cardiaca sistólica } \\
\text { que no requerían } \\
\text { inotrópico Iv. } 92 \% \text { NYHA } \\
\text { basal III-IV, media de FE } \\
23 \%(n=949)\end{array}$ & $\begin{array}{l}\text { Milrinone en infusión por } 48 \\
\text { horas } 0,5 \mathrm{mcg} / \mathrm{kg} / \mathrm{min} \\
(\mathrm{n}=477)\end{array}$ & Placebo $(n=472)$ & 60 días & $\begin{array}{l}\text { Muerte } \\
\text { intrahospitalaria: } \\
\text { Milrinone }=18 / 477 \\
\text { vs. } \\
\text { placebo }=11 / 472\end{array}$ \\
\hline
\end{tabular}


selectivo de resultados [(Packer (2003 y 2013) (REVIVE I), Colettaa (2004), Cleland (2004), Zairis (2004) - CASINO, Cleland (2006 y Packer (2013) (REVIVE ॥) y Samimi (2008)]. En 3 de ellos [(Packer (2003 y 2013) (REVIVE I), Colettaa (2004), Cleland (2004), Zairis (2004) - CASINO Cleland (2006 y Packer (2013) (REVIVE ॥)] se encontraron diferencias entre varias publicaciones del mismo estudio y en otro [Samimi (2008)] el desenlace no se había planteado en la publicación inicial. Finalmente, 4 estudios [(Nieminen (2000), Colettaa (2004), Cleland (2004), Zairis (2004) - CASINO, Bergh (2010) y Cuffe (2002)] se consideraron con alto riesgo de otros sesgos. En 2 de ellos [(Nieminen (2000), y Cuffe (2002) OPTIME)] solo se incluyeron pacientes con falla cardiaca estable 0 se excluyeron aquellos con indicación de soporte vasopresor o inotrópico, 1 [Bergh (2010)] fue suspendido antes de reclutar el tamaño de muestra planeado y el otro [(Colettaa (2004), Cleland (2004), Zairis (2004)] fue suspendido de manera prematura por beneficio.

En conclusión, la mayoría de estudios mostró riesgo de sesgos de selección de la población y enmascaramiento de la intervención. Solo 3 estudios [(Husebye (2013) LEAF, Follath (2002) LIDO, Mebazaa (2007) SURVIVE)] se consideraron con bajo riesgo de sesgos mientras otros 3 con alto riesgo de sesgos [(Packer (2003 y 2013) (REVIVE I) Kivikko (2003), Colettaa (2004), Cleland (2004), Zairis (2004) - CASINO, Cleland (2006 y Packer (2013) (REVIVE ॥)] (tabla 2).

\section{Síntesis cuantitativa de los estudios}

Se hicieron comparaciones directas de los tres tratamientos activos contra placebo, dos comparaciones directas de tratamientos activos cabeza-cabeza (levosimendándobutamina y dobutamina-milrinone) y una comparación indirecta (levosimendán-milrinone) (tabla 3).

La comparación de los tratamientos debe ser leída de izquierda a derecha y su correspondiente OR está en la celda respectiva que cruza la columna con la fila.

No se demostraron diferencias significativas en el desenlace evaluado entre los diversos tratamientos ni contra placebo ni entre ellos, excepto cuando se comparó levosimendán contra dobutamina, en el que hay reducción del $34 \%$ del riesgo de muerte; sin embargo, el efecto se pierde al evaluar los intervalos de predicción, por lo que la conclusión no cambia. Los rangos de los intervalos de confianza y de predicción son bastante amplios, a pesar del aumento del número de pacientes con la combinación de estudios (fig. 3).

El análisis por rangos de la probabilidad de clasificación acumulada (SUCRA) demuestra que entre los tratamientos evaluados el milrinone muestra la mayor probabilidad de ser el mejor (80\%), seguido por la dobutamina (70\%) (fig. 4).

No se demostró inconsistencia en el modelo global, en las comparaciones realizadas $\left(c h i^{2}=6,61 ; p=0,15\right)$.

\section{Discusión}

El principal hallazgo de este metaanálisis es que la utilización de medicamentos inotrópicos como levosimendán, dobutamina o milrinone no demuestra ningún beneficio sobre la mortalidad en pacientes con falla cardiaca aguda con fracción de eyección reducida al compararse con placebo o entre ellos, aunque se debe tener en cuenta la heterogeneidad clínica detectada entre los ensayos

Tabla 2 Clasificación del riesgo de sesgos en los estudios incluidos

\begin{tabular}{|c|c|c|c|c|c|c|}
\hline Estudio & $\begin{array}{l}\text { Generación } \\
\text { secuencia } \\
\text { asignación }\end{array}$ & $\begin{array}{l}\text { Ocultamiento } \\
\text { de la } \\
\text { asignación }\end{array}$ & Cegamiento & $\begin{array}{l}\text { Desenlaces } \\
\text { incompletos }\end{array}$ & $\begin{array}{l}\text { Reporte } \\
\text { selectivo }\end{array}$ & $\begin{array}{l}\text { Otros } \\
\text { sesgos }\end{array}$ \\
\hline Nieminen (2000) & No claro & No claro & No claro & Bajo & Bajo & Alto \\
\hline Moiseyev (2002) & Bajo & No claro & Bajo & Bajo & Bajo & Bajo \\
\hline Packer (2003 y 2013) (REVIVE ।) & No claro & No claro & No claro & Bajo & Alto & Bajo \\
\hline Kivikko (2003) & No claro & No claro & No claro & Bajo & Bajo & Bajo \\
\hline $\begin{array}{l}\text { Colettaa (2004), Clelanda (2004), } \\
\text { Zairis (2004) - CASINO }\end{array}$ & No claro & No claro & No claro & Bajo & Alto & Alto \\
\hline $\begin{array}{l}\text { Cleland (2006 y Packer (2013) } \\
\text { (REVIVE ॥) }\end{array}$ & No claro & No claro & No claro & Bajo & Alto & Bajo \\
\hline Adamopoulos (2006) & No claro & No claro & No claro & Bajo & Bajo & Bajo \\
\hline Flevari (2006) & No claro & No claro & No claro & Bajo & Bajo & Bajo \\
\hline Husebye (2013) LEAF & Bajo & Bajo & Bajo & Bajo & Bajo & Bajo \\
\hline Follath (1999) & No claro & No claro & No claro & Bajo & Bajo & Bajo \\
\hline Follath (2002) LIDO & Bajo & Bajo & Bajo & Bajo & Bajo & Bajo \\
\hline Samimi (2008) & No claro & Alto & No claro & Bajo & Alto & Bajo \\
\hline Duygu (2007) & No claro & Alto & No claro & Bajo & Bajo & Bajo \\
\hline Duygu (2008) & No claro & No claro & No claro & Bajo & Bajo & Bajo \\
\hline Mebazaa (2007) SURVIVE & Bajo & Bajo & Bajo & Bajo & Bajo & Bajo \\
\hline Bergh (2010) & No claro & No claro & No claro & Bajo & Bajo & Alto \\
\hline Liang (2014) & No claro & No claro & No claro & Bajo & Bajo & Bajo \\
\hline Bader (2010) & No claro & Alto & No claro & Bajo & Bajo & Bajo \\
\hline Cuffe (2002) OPTIME & Bajo & Bajo & Bajo & Bajo & Bajo & Alto \\
\hline Aranda (2003) & No claro & Alto & No claro & Bajo & Bajo & Bajo \\
\hline
\end{tabular}


Tabla 3 Efecto sobre la mortalidad de los diferentes tratamientos (tabla de liga)

\begin{tabular}{|c|c|c|c|}
\hline Placebo & $\begin{array}{l}1,30(0,80-2,11) \\
\text { Dobutamina } \\
0,66(0,45-0,97) \\
1,30(0,45-3,72)\end{array}$ & $\begin{array}{l}0,86(0,58-1,28) \\
\text { Levosimendán } \\
1,97(0,71-5,46)\end{array}$ & $1,69(0,65-4,36)$ \\
\hline
\end{tabular}

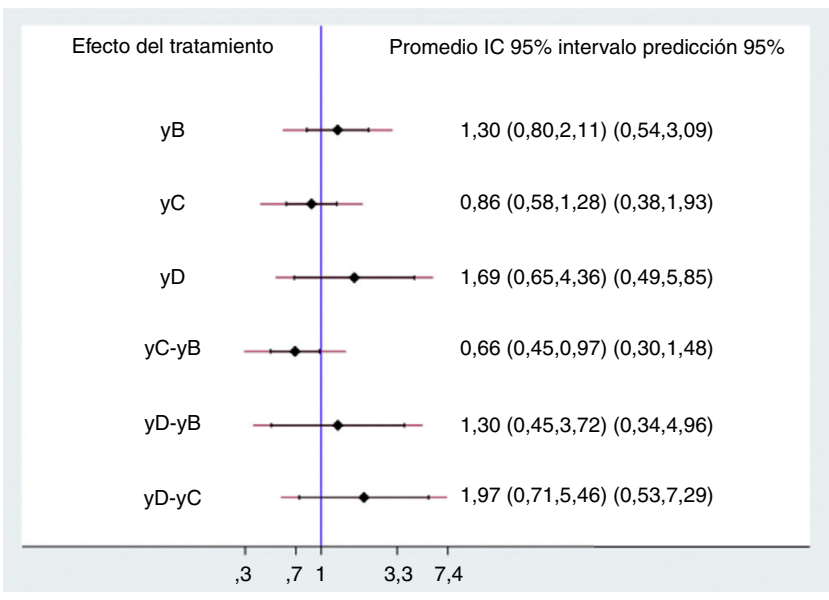

Figura 3 Comparaciones directas e indirectas de inotrópicos. Desenlace muerte. $A=$ placebo; $B=$ dobutamina; $C=$ levosimendán; $D=$ milrinone. Se expresan los OR con su IC 95\% (línea negra) y el correspondiente intervalo de predicción 95\% (línea extendida roja) entre los diversas comparaciones.

incluidos; se demostró menor mortalidad al comparar levosimendán contra dobutamina (OR 0,66 IC 95\% 0,45-0,97); no obstante, la significancia desaparece al utilizar los intervalos de predicción (IP 95\% 0,30-1,48). El intervalo de predicción es un concepto diferente al del intervalo de confianza en la estadística frecuentista y al intervalo de credibilidad en la estadística bayesiana; el intervalo de confianza está asociado con un parámetro y predice la distribución del estadístico, el intervalo de credibilidad se asocia con la posterior distribución del parámetro y el intervalo de predicción es el estimado de un intervalo en el cual caerán futuras observaciones, o sea, el rango de futuros ensayos.

Hasta el momento no son muchos los metaanálisis publicados que evalúen el efecto de los inotrópicos sobre la mortalidad en este grupo de pacientes, especialmente con el levosimendán como principal intervención ${ }^{33}$, aunque sigue siendo escasa la información en cuanto a comparaciones con diseño de metaanálisis en red $^{34}$. Los metaanálisis disponibles poseen dificultades metodológicas, básicamente por combinar los escenarios de utilización del medicamento (cirugía cardiovascular, falla cardiaca aguda, sepsis), lo que se conoce como mezclar "peras con manzanas" (manzanas con naranjas) ${ }^{35-37}$, inclusión de estudios retractados posteriormente ${ }^{36,38}$ y la incertidumbre de los resultados del estudio CASINO ${ }^{15,39-42}$.

Este último fue un ensayo con asignación aleatoria a tres grupos de tratamiento: uno con levosimendán, otro con dobutamina y otro con placebo, en el que se buscó evaluar la mortalidad a 1 y 6 meses. Estaba planeado para incluir 600 pacientes, pero se suspendió en forma temprana cuando tenían una muestra de 299 pacientes (100 en el grupo de

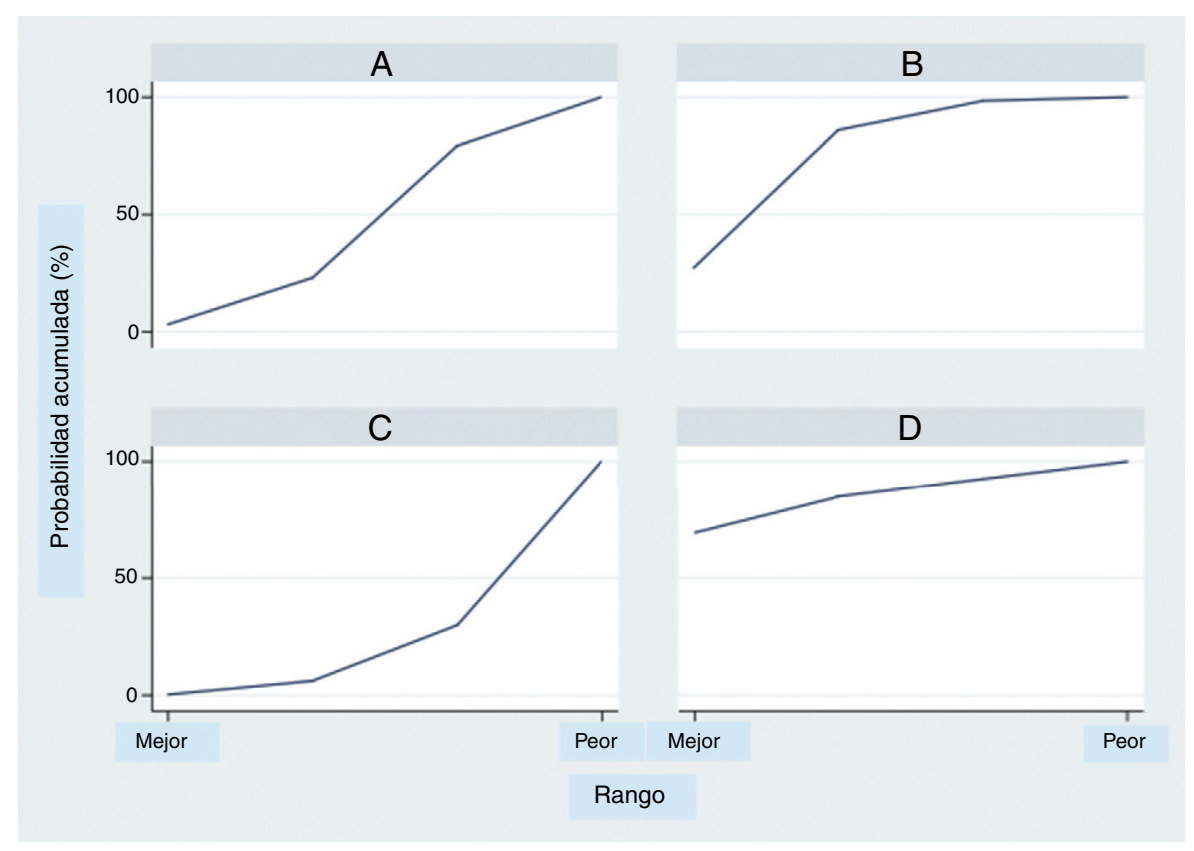

Figura 4 Probabilidad de clasificación acumulada con base en el rango por área bajo la curva. A=placebo; B=dobutamina; $\mathrm{C}=$ levosimendán; $\mathrm{D}=$ milrinone. 
levosimendán, 100 en el de dobutamina y 99 en el placebo), debido a diferencias marcadas en mortalidad que favorecían al levosimendán; se encontró según lo reportado, mortalidad a 1 mes de $6,1 \%$ con levosimendán, $12,8 \%$ con dobutamina y $8,2 \%$ para placebo ( $p=0,1$ vs. placebo y 0,04 vs. dobutamina); y mortalidad a 6 meses de $15,3 \%$ con levosimendán, $39,6 \%$ con dobutamina y $24,7 \%$ para placebo ( $p=0,0001$ vs. dobutamina y 0,04 vs. placebo); sin embargo, el estudio solo se publicó en formato de resumen, por lo cual se desconocen detalles de su diseño, incluyendo los parámetros definidos para la suspensión temprana con solo la mitad de la población calculada inicialmente, hecho que puede afectar los resultados ${ }^{43}$, y discrepancias en los resúmenes disponibles, dado que en una publicación se menciona que se extrajeron 8 pacientes del análisis, pero en la siguiente se reporta el dato de $100 \%$ de seguimiento.

Infortunadamente, esta no ha sido la excepción en los grandes estudios con levosimendán, puesto que los resultados completos de los estudios REVIVE se conocieron mucho tiempo después de su terminación ${ }^{44}$. En los REVIVE । y II se evaluaron los cambios en el estado clínico en los primeros 5 días después de la aleatorización (se evaluaban a las 6 y 24 horas y a los 5 días), definidos como mejoría, sin cambio o empeoramiento ${ }^{18}$. En el REVIVE I se encontró que numéricamente más pacientes del grupo de levosimendán mejoraron y menos empeoraron en comparación con el placebo, tanto a 6 y 24 horas como a 5 días, pero este hallazgo no fue estadísticamente significativo $(p=0,134$ para todas las diferencias entre los grupos). En el REVIVE ॥ las diferencias siempre fueron significativas a favor de levosimendán $(p=0,015$ con criterios estrictos y $<0,001$ con criterios menos estrictos para todas las diferencias entre los grupos). En cuanto a la mortalidad total a 90 días, si bien numéricamente se presentó una cifra mayor en los pacientes asignados al grupo de levosimendán, no fue estadísticamente significativa, con un HR de 1,26 (IC: $0,83-1,91$ ) y $p=0,29$ para ambos estudios combinados $^{18}$.

A pesar de la evidencia que demuestra pobre impacto sobre la mortalidad y el aumento de algunos efectos secundarios potencialmente peligrosos, los inotrópicos son necesarios en el manejo de pacientes con falla cardiaca aguda con reducción severa del gasto cardiaco que produce hipoperfusión generalmente con hipotensión, siempre y cuando se haya descartado hipovolemia u otras causas corregibles $^{1,45}$. Los inodilatadores producen aumentos variables del gasto cardiaco y reducción de la resistencia vascular periférica y de la presión en cuña de la arteria pulmonar ${ }^{46-48}$. Los datos hemodinámicos favorecen el efecto del levosimendán y del milrinone, especialmente en presencia del efecto de betabloqueadores ${ }^{49,50}$. Sin embargo, se debe tener precaución con el uso de milrinone en pacientes con cardiopatía isquémica, puesto que un subanálisis del estudio OPTIME CHF demostró mayor mortalidad con su uso, en ese grupo específico ${ }^{51}$.

El uso del análisis por rangos de la probabilidad de clasificación acumulada (SUCRA) debe hacerse con precaución, ya que aunque es de más fácil acceso para el clínico, posee algunos problemas importantes al interpretarlos ${ }^{52}$. Esta evaluación puede ser inestable por los supuestos estadísticos $^{53}$, se afectan por el número de tratamientos y estudios incluidos ${ }^{54}$ y por la estructura de la red ${ }^{55}$; por lo tanto, aunque el presente análisis demuestra una probabilidad mayor de ser el mejor tratamiento para el milrinone seguido por la dobutamina y en último lugar el levosimendán, debe interpretarse con cautela, máxime cuando no se demostraron diferencias estadísticamente significativas entre los tres tratamientos evaluados.

\section{Limitaciones}

La mayor limitación, inherente a todos los metaanálisis publicados hasta el momento en este escenario, es la inevitable heterogeneidad clínica observada en los estudios incluidos, aunque se pueda presumir transitividad dadas las características de la población. Para controlarlo se incluyeron solamente los estudios en falla cardiaca aguda, con fracción de eyección reducida, que reportaron el desenlace de interés, y solo en las comparaciones definidas. No fue posible hacer análisis de otros desenlaces, incluyendo los de seguridad, por inadecuado reporte en los ensayos clínicos. Medicamentos con eficacia limitada o pequeñas diferencias entre los tratamientos podrían, en teoría, requerir un número mayor de participantes en los estudios y en el global. Hay una diferencia marcada en el año de inicio de muchos de estos estudios que implica cambios tecnológicos y de tratamiento concomitante utilizados en ellos, que pueden influir en los resultados. A pesar de estas consideraciones, era importante establecer una aproximación que generara la menor incertidumbre posible para la toma de decisiones en la práctica diaria y sustentara una recomendación emitida por una guía de práctica clínica en la pregunta considerada, en la cual toma relevancia el aspecto de costo-efectividad.

\section{Conclusiones}

En pacientes con falla cardiaca aguda no hay diferencias en el uso de inotrópicos como el levosimendán, la dobutamina o el milrinone, en términos de reducción del desenlace de mortalidad. Por lo tanto, su indicación dependerá de otros aspectos como hipoperfusión, características basales de los pacientes y costo-efectividad de cada uno de ellos.

\section{Financiación}

Grupo para la Investigación de las Enfermedades Cardiovasculares.

\section{Responsabilidades éticas}

Protección de personas y animales. Los autores declaran que para esta investigación no se han realizado experimentos en seres humanos ni en animales.

Confidencialidad de los datos. Los autores declaran que en este artículo no aparecen datos de pacientes.

Derecho a la privacidad y consentimiento informado. Los autores declaran que en este artículo no aparecen datos de pacientes. 


\section{Conflictos de interés y aspectos éticos}

La investigación fue financiada con recursos propios del Grupo para la Investigación de las Enfermedades Cardiovasculares, de la sección de Cardiología de la Universidad de Antioquia y no hay conflictos de interés por declarar.

Los aspectos éticos se ajustan a los principios éticos fundamentales y a las directrices de la Declaración de Helsinki y similares, emanados de la Asociación Médica Mundial, y sigue las pautas del Ministerio de la Protección Social de la República de Colombia según la resolución 8430 de 1993 por la cual se dictan las normas científicas, técnicas y administrativas para la investigación en salud.

\section{Bibliografía}

1. Ponikowski P, Voors AA, Anker SD, Bueno H, Cleland JG, Coats AJ, et al. 2016 ESC Guidelines for the diagnosis and treatment of acute and chronic heart failure: The Task Force for the diagnosis and treatment of acute and chronic heart failure of the European Society of Cardiology (ESC)Developed with the special contribution of the Heart Failure Association (HFA) of the ESC. Eur Heart J. 2016;37:2129-200, http://dx.doi.org/10.1093/ eurheartj/ehw128.

2. Gheorghiade M, Zannad F, Sopko G, Klein L, Piña IL, Konstam MA, et al. Acute heart failure syndromes: current state and framework for future research. Circulation. 2005;112:3958-68.

3. Adams KF Jr, Fonarow GC, Emerman CL, LeJemtel TH, Costanzo MR, Abraham WT, et al. Characteristics and outcomes of patients hospitalized for heart failure in the United States: rationale, design, and preliminary observations from the first 100,000 cases in the Acute Decompensated Heart Failure National Registry (ADHERE). Am Heart J. 2005;149:209-16.

4. Yancy CW, Lopatin M, Stevenson LW, De Marco T, Fonarow GC, ADHERE Scientific Advisory Committee and Investigators. Clinical presentation, management, and in-hospital outcomes of patients admitted with acute decompensated heart failure with preserved systolic function: a report from the Acute Decompensated Heart Failure National Registry (ADHERE) Database. J Am Coll Cardiol. 2006;47:76-84.

5. Belletti A, Castro ML, Silvetti S, Greco T, Biondi-Zoccai G, Pasin $L$, et al. The Effect of inotropes and vasopressors on mortality: a meta-analysis of randomized clinical trials. $\mathrm{Br} \mathrm{J}$ Anaesth. 2015;115:656-75, http://dx.doi.org/10.1093/bja/aev284.

6. Francis G, Bartos JA, Adatya S. Inotropes. J Am Coll Cardiol. 2014;63:2069-78, http://dx.doi.org/10.1016/j.jacc. 2014.01.016.

7. Gong B, Li Z, Yat Wong PC. Levosimendan treatment for heart failure: a systematic review and meta-analysis. J Cardiothorac Vasc Anesth. 2015;29:1415-25, http://dx.doi.org/10.1053/ j.jvca.2015.03.023.

8. Greco T, Zangrillo A, Biondi-Zocai G, Landoni G. Meta-analysis: pitfalls and hints. Heart, Lung and Vessels. 2013;5:219-25.

9. Li T, Puhan MA, Vedula SS, Singh S, Dickersin K, and The Ad Hoc Network Meta-analysis Methods Meeting Working Group. Network meta-analysis-highly attractive but more methodological research is needed. BMC Medicine. 2011;9:79 DOI: 10.1186/1741-7015-9-79.

10. Greco T, Biondi-Zocai G, Saleh O, Pasin L, Cabrini L, Zangrillo A, Landoni G. The attractiveness of network meta-analysis: a comprehensive systematic and narrative review. Heart, Lung and Vessels. 2015;7:133-42.

11. Liberati A, Altman DG, Tetzlaff J, Mulrow C, Gøtzsche PC, loannidis JP, et al. The PRISMA statement for reporting systematic reviews and meta-analyses of studies that evaluate health care interventions: explanation and elaboration. Ann Intern Med. 2009;151, http://dx.doi.org/10.7326/ 0003-4819-151-4-200908180-00136. W65-94.

12. Hutton B, Salanti G, Caldwell DM, Chaimani A, Schmid $\mathrm{CH}$, Cameron $\mathrm{C}$, et al. The PRISMA extension statement for reporting of systematic reviews incorporating network meta-analyses of health care interventions: checklist and explanations. Ann Intern Med. 2015;162:777-84, http://dx.doi.org/10.7326/M14-2385.

13. Nieminen MS, Akkila J, Hasenfuss G, Kleber FX, Lehtonen LA, Mitrovic V, et al. Hemodynamic and neurohumoral effects of continuous infusion of levosimendan in patients with congestive heart failure. J Am Coll Cardiol. 2000;36:1903-12.

14. Adamopoulos S, Parissis JT, Iliodromitis EK, Paraskevaidis I, Tsiapras D, Farmakis D, et al. Effects of levosimendan versus dobutamine on inflammatory and apoptotic pathways in acutely decompensated chronic heart failure. Am J Cardiol. 2006;98:102-6.

15. Cleland JG, Ghosh J, Freemantle N, Kaye GC, Nasir M, Clark AL, Coletta AP. Clinical trials update and cumulative meta-analyses from the American College of Cardiology: WATCH, SCD-HeFT, DINAMIT, CASINO, INSPIRE, STRATUS-US, RIO-Lipids and cardiac resynchronisation therapy in heart failure. Eur $\mathrm{J}$ Heart Fail. 2004;6:501-8.

16. Moiseyev VS, Põder P, Andrejevs N, Ruda MY, Golikov AP, Lazebnik LB, et al. Safety and efficacy of a novel calcium sensitizer, levosimendan, in patients with left ventricular failure due to an acute myocardial infarction. A randomized, placebocontrolled, double-blind study (RUSSLAN). Eur Heart J. 2002;23: 1422-32.

17. Packer M, Colucci W, Fisher L, Massie B, Teerlink JR, Young JB, et al. Development of a comprehensive new endpoint for the evaluation of new treatments for acute decompensated heart failure: results with levosimendan in the REVIVE 1 study. J Card Fail. 2001;9:\$61.

18. Packer M, Colucci W, Fisher L, Massie BM, Teerlink JR, Young $\mathrm{J}$, et al. Effect of levosimendan on the short-term clinical course of patients with acutely decompensated heart failure. JACC Heart Fail. 2013;1:103-11, http://dx.doi.org/ 10.1016/j.jchf.2012.12.004.

19. Flevari P1, Parissis JT, Leftheriotis D, Panou F, Kourea K, Kremastinos DT. Effect of levosimendan on ventricular arrhythmias and prognostic autonomic indexes in patients with decompensated advanced heart failure secondary to ischemic or dilated cardiomyopathy. Am J Cardiol. 2006;98:1641-5.

20. Husebye T, Eritsland J, Müller C, Sandvik L, Arnesen H, Seljeflot $\mathrm{I}$, et al. Levosimendan in acute heart failure following primary percutaneous coronary intervention-treated acute STelevation myocardial infarction. Results from the LEAF trial: a randomized, placebo-controlled study. Eur J Heart Fail. 2013;15:565-72, http://dx.doi.org/10.1093/eurjhf/hfs215.

21. Folláth F, Hinkka S, Jager D, Just H, Mitrovic V, Papp JG, et al. Dose-ranging and safety with intravenous levosimendan in lowoutput heart failure: experience in three pilot studies and outline of the Levosimendan Infusion Versus DObutamine (LIDO) Trial. Am J Cardiol. 1999, 83:21(I)-25(I).

22. Follath F, Cleland JG, Just H, Papp JG, Scholz H, Peuhkurinen $\mathrm{K}$, et al. Efficacy and safety of intravenous levosimendan compared with dobutamine in severe low-output heart failure (the LIDO study): a randomised double-blind trial. Lancet. 2002;360:196-202.

23. Samimi-Fard S, García-González MJ, Domínguez-Rodríguez A, Abreu-González P. Effects of levosimendan versus dobutamine on long-term survival of patients with cardiogenic shock after primary coronary angioplasty. Int J Cardiol. 2008;127: 284-7.

24. Duygu H, Nalbantgil S, Ozerkan F, Zoghi M, Akilli A, Erturk U, et al. Effects of levosimendan on left atrial functions in patients 
with ischemic heart failure. Clin Cardiol. 2008;31:607-13, http://dx.doi.org/10.1002/clc.20332.

25. Mebazaa A1, Nieminen MS, Packer M, Cohen-Solal A, Kleber FX, Pocock SJ, et al. Levosimendan vs dobutamine for patients with acute decompensated heart failure: the SURVIVE Randomized Trial. JAMA. 2007;297:1883-91.

26. Duygu H, Turk U, Ozdogan O, Akyuz S, Kirilmaz B, Alioglu $E$, et al. Levosimendan versus dobutamine in heart failure patients treated chronically with carvedilol. Cardiovasc Ther. 2008;26:182-8, http://dx.doi.org/10.1111/ j.1755-5922.2008.00050.x

27. Bergh CH, Andersson B, Dahlström U, Forfang K, Kivikko $M$, Sarapohja T, et al. Intravenous levosimendan vs. dobutamine in acute decompensated heart failure patients on beta-blockers. Eur J Heart Fail. 2010;12:404-10, http://dx.doi.org/10.1093/eurjhf/hfq032.

28. Liang CS, Sherman LG, Doherty JU, Wellington K, Lee VW, Hood WB Jr. Sustained improvement of cardiac function in patients with congestive heart failure after short-term infusion of dobutamine. Circulation. 1984;69:113-9.

29. Bader FM, Gilbert EM, Mehta NA, Bristow MR. Double-blind placebo-controlled comparison of enoximone and dobutamine infusions in patients with moderate to severe chronic heart failure. Congest Heart Fail. 2010;16:265-70, http://dx.doi.org/10.1111/j.1751-7133.2010.0018.5.x.

30. Cuffe MS, Califf RM, Adams KF Jr, Benza R, Bourge R, Colucci WS, et al. Short-term intravenous milrinone for acute exacerbation of chronic heart failure: a randomized controlled trial. JAMA. 2002;287:1541-7.

31. Aranda JM Jr, Schofield RS, Pauly DF, Cleeton TS, Walker TC, Monroe VS Jr, et al. Comparison of dobutamine versus milrinone therapy in hospitalized patients awaiting cardiac transplantation: a prospective, randomized trial. Am Heart J. 2003;145:324-9.

32. Kivikko M, Lehtonen L, Colucci WS. Sustained hemodynamic effects of intravenous levosimendan. Circulation. 2003;107:81-6.

33. Pollesello P, Parissis J, Kivikko M, Harjola VP. Levosimendan meta-analyses: Is there a pattern in the effect on mortality? Int J Cardiol. 2016;209:77-83, http://dx.doi.org/10.1016/ j.ijcard.2016.02.014.

34. Greco T, Calabrò MG, Covello RD, Greco M, Pasin L, Morelli A, et al. A Bayesian network meta-analysis on the effect of inodilatory agents on mortality. $\mathrm{Br} \mathrm{J}$ Anaesth. 2015;114:746-56, http://dx.doi.org/10.1093/bja/aeu446.

35. Meco M, Cirri S. Can a meta-analysis that mixes apples with oranges be used to demonstrate that levosimendan reduces mortality after coronary revascularization? Crit Care. 2011;15:455, http://dx.doi.org/10.1186/cc10484, author reply 455.

36. Landoni G, Biondi-Zoccai G, Greco M, Greco T, Bignami E, Morelli A, et al. Effects of levosimendan on mortality and hospitalization. A meta-analysis of randomized controlled studies. Crit Care Med. 2012;40:634-46, http://dx.doi.org/ 10.1097/CCM.0b013e318232962a.

37. Huang X, Lei S, Zhu MF, Jiang RL, Huang LQ, Xia GL, Zhi YH. Levosimendan versus dobutamine in critically ill patients: a metaanalysis of randomized controlled trials. J Zhejiang Univ Sci B. 2013;14:400-15, http://dx.doi.org/10.1631/jzus.B1200290.

38. De Hert SG, Lorsomradee S, vanden Eede H, Cromheecke S, Van der Linden PJ. A randomized trial evaluating different modalities of levosimendan administration in cardiac surgery patients with myocardial dysfunction. J Cardiothorac Vasc Anesth. 2008;22:699-705. Retraction: J Cardiothorac Vasc Anesth. 2011;25:897.

39. Zairis M, Apostolatos C, Anastasiadis P, Mytas D, Katsaris C, Kouris $\mathrm{H}$, et al. The effect of a calcium sensitizer or an inotrope in chronic low outpout decompensated heart failure: results from the calcium sensitizer or inotrope or none in low output heart failure study (CASINO). J Am Coll Cardiol. 2004;43(5s1):835-6.

40. Coletta AP, Cleland JGF, Freemantle N, Clark AL. Clinical trials update from the European Society of Cardiology Heart Failure meeting: SHAPE, BRING-UP 2 VAS, COLA II, FOSIDIAL, BETACAR, CASINO and meta-analysis of cardiac resynchronisation therapy. Eur J Heart Fail. 2004;6:673-6.

41. Delaney A, Bradford C, McCaffrey J, Bagshaw SM, Lee R. Levosimendan for the treatment of acute severe heart failure: a meta-analysis of randomised controlled trials. Int J Cardiol. 2010;138:281-9, http://dx.doi.org/10.1016/ j.ijcard.2008.08.020.

42. Ribeiro RA, Rohde LE, Polanczyk CA. Levosimendan in acute decompensated heart failure: systematic review and metaanalysis. Arq Bras Cardiol. 2010;95:230-7.

43. Bassler D, Montori VM, Briel M, Glasziou P, Guyatt G. Early stopping of randomized clinical trials for overt efficacy is problematic. J Clin Epidemiol. 2008;61:241-6, http://dx.doi.org/10.1016/j.jclinepi.2007.07.016.

44. Califf RM. Better late than never: a welcome publication of tardy clinical trial results. JACC Heart Fail. 2013;1:112-4, http://dx.doi.org/10.1016/j.jchf.2013.02.001.

45. Goldhaber JI, Hamilton MA. Role of inotropic agents in the treatment of heart failure. Circulation. 2010;121:1655-60, http://dx.doi.org/10.1161/CIRCULATIONAHA.109.899294.

46. Akhtar N, Mikulic E, Cohn JN, Chaudhry MH. Hemodynamic effect of dobutamine in patients with severe heart failure. Am J Cardiol. 1975;36:202-5.

47. Moertl D, Berger R, Huelsmann M, Bojic A, Pacher R. Short-term effects of levosimendan and prostaglandin E1 on hemodynamic parameters and B-type natriuretic peptide levels in patients with decompensated chronic heart failure. Eur J Heart Fail. 2005; 7:1156-63.

48. Givertz MM, Hare JM, Loh E, Gauthier DF, Colucci WS. Effect of bolus milrinone on hemodynamic variables and pulmonary vascular resistance in patients with severe left ventricular dysfunction: a rapid test for reversibility of pulmonary hypertension. J Am Coll Cardiol. 1996;28:1775-80.

49. Mebazaa A, Nieminen MS, Filippatos GS, Cleland JG, Salon JE, Thakkar R, et al. Levosimendan vs. dobutamine: outcomes for acute heart failure patients on (-blockers in SURVIVE. Eur J Heart Fail. 2009;11:304-11, http://dx.doi.org/10.1093/ eurjhf/hfn045.

50. Travill CM, Pugh S, Noble MI. The inotropic and hemodynamic effects of intravenous milrinone when reflex adrenergic stimulation is suppressed by beta-adrenergic blockade. Clin Ther. 1994; 16:783-92.

51. Felker GM, Benza RL, Chandler AB, Leimberger JD, Cuffe MS, Califf RM, Gheorghiade M, et al. Heart failure etiology and response to milrinone in decompensated heart failure: results from the OPTIME-CHF study. J Am Coll Cardiol. 2003;41:997-1003.

52. Trinquart L, Attiche N, Bafeta A, Porcher R, Ravaud P. Uncertainty in treatment rankings: reanalysis of network meta-analyses of randomized trials. Ann Intern Med. 2016;164:666-73, http://dx.doi.org/10.7326/M15-2521.

53. van Valkenhoef $\mathrm{G}$, Ades AE. Evidence synthesis assumes additivity on the scale of measurement: response to "Rank reversal in indirect comparisons" by Norton et al. [Letter]. Value Health. 2013;16:449-51, http://dx.doi.org/ 10.1016/j.jval.2012.11.012.

54. Mills EJ, Kanters S, Thorlund K, Chaimani A, Veroniki AA, Ioannidis JP. The effects of excluding treatments from network metaanalyses: survey. BMJ. 2013;347:f5195, http://dx.doi.org/ 10.1136/bmj.f5195.

55. Rucker G, Schwarzer G. Ranking treatments in frequentist network meta-analysis works without resampling methods. BMC Med Res Methodol. 2015;15:58, http://dx.doi.org/ 10.1186/s12874-015-0060-8. 